\title{
NEW INSIGHTS INTO SOLAR WIND IMPLANTED VOLATILES FOR LUNAR REGOLITH CHARACTERIZATION: A SIMULATION BASED APPROACH
}

\author{
Shashwat Shukla ${ }^{1, *}$, Sayantan Majumdar ${ }^{1}$, Abhisek Maiti ${ }^{1}$, Shashi Kumar ${ }^{2}$ \\ ${ }^{1}$ Department of Geo-Informatics, Indian Institute of Remote Sensing, Indian Space Research Organization, Dehradun, India - \\ (shukla, s.majumdar, maiti)@student.utwente.nl \\ ${ }^{2}$ Photogrammetry and Remote Sensing Department, Indian Institute of Remote Sensing, Indian Space Research Organization, \\ Dehradun, India - shashi@iirs.gov.in
}

\section{Commission IV, WG IV/3}

KEY WORDS: Solar wind implanted volatiles, ICME, Activation energy, Regolith, Exosphere, Simulation, Solar Astronomy

\begin{abstract}
:
The effect of solar wind implanted volatiles into the top $100 \mathrm{~nm}$ of the lunar regolith plays a significant role in quantitatively assessing the lunar surface isotopic composition. In essence, these volatiles can either quickly sputter out of the surface or be retained. The implantation processes exhibit a functional dependency on the surface temperature, ilmenite abundance and the activation energy associated with the optical maturity of the lunar soil. The prime focus of this study is to simulate the implication of these incident volatiles in characterizing the regolith for a better insight into the modeling of lunar exosphere during both Interplanetary Coronal Mass Ejection (ICME) and usual cases. Additionally, the proposed model quantifies the total lunar oxygen repository along with determining the associated textural and frequency domain measures for probable future lunar ${ }^{3} \mathrm{He}$ mining sites. In this 30 -day simulation, the particles bombard the reconstructed lunar grid wherein each cell displays varying particle density at a given local time. Moreover, both the activation energy and $\mathrm{TiO}_{2}$ content are assumed to be in a Gaussian distribution having $\left(\mu, \sigma^{*}\right)$ of $(0.96,0.025)$ and $(12.52,3.44)$ respectively. It has been found that the surfaces characterized by high activation energy tend to retain solar wind implants due to the large numbers of crystal defects. However, for $\mathrm{H}$ and heavy trace ions, intermediate activation energy range demonstrates diurnal behavior with the diffusive loss at local noon time. The study also finds an intriguing relationship between the lunar $\mathrm{O}_{2}$ and retained $\mathrm{H}$ sites (frequency domain). Furthermore, this could be utilized as a generic exospheric modeling paradigm for airless bodies and contribute to the understanding of the physical processes associated with solar astronomy.
\end{abstract}

\section{INTRODUCTION}

The interaction of solar wind with the local planetary surfaces provides an insight into the implantation processes of the volatiles carried out by the wind. It serves as a characteristic attribute to the evolution of the stellar system (in this case, the Sun), by affecting the exospheres of the celestial bodies. Compositionally, this energized stream of charged particles comprises of mainly protons along with trace proportions of heavy elements like $\mathrm{O}^{7+},{ }^{3} \mathrm{He}$, etc. (Killen, Hurley, \& Farrell, 2012). The major source of solar wind is plasma, which arises from hot solar corona expanding into space at speeds nominally near $400 \mathrm{Km} / \mathrm{s}$. However, during the time of Interplanetary Coronal Mass Ejection (ICME), the speed and density of the solar wind get intensified. Several observations of the upper atmosphere of the Earth quantitatively assessed the correlation between the solar flares and appearance of Aurora including terrestrial magnetic storms (Schoröder, 2008). Furthermore, recent measurements by the Sub-KeV Atom Reflecting Analyser (SARA) instrument onboard Chandrayaan -1 reported the penetration depth of $\mathrm{H}^{+}$(protons) into the lunar grains to be 5 $10 \mathrm{~nm}$ (Wieser et al., 2009). For a while, it has been widely accepted that the illuminated lunar surface absorbs the entire incident solar wind plasma until Wieser et al., 2009 quantified the assessment of reflected solar wind in the form of neutral hydrogen atoms (protons) using SARA instrument (Feldman, 1998; Schmitt, Kulcinski, Santarius, Ding, Malecki, \& Zalewski, 2000; Wieser et al., 2009). The study also measured the amount of impinging solar wind protons that were reflected back to space and found an intriguing 20\% neutral atomic hydrogen reflections from the surface (Wieser et al., 2009).
In addition to this, the lunar exosphere serves as a source of neutrals which are then subjected to ionization by protons, electrons and photons (Cladis, Francis, \& Vondrak, 1994; Winske, Wu, Li, Mou, \& Guo, 1985). These ionized particles influence the interaction of solar wind with the lunar surface through momentum transfer while they get accelerated by the motional electric field. Farrell et al., 2012 investigated the entire lunar surface, exosphere, and solar wind plasma system during a space weather event using the Solar-Storm Lunar Atmosphere Modelling (SSLM). During the interaction of the solar wind with the surface, the volatiles impacts the regolith based on the nature of lunar soil grains at the molecular level.

\subsection{Effects of Space Weathering on Lunar Soil}

A layer of unconsolidated crushed rocks, glass, and minerals constitute the lunar regolith (Heiken, Vaniman, \& French, 1991). The formation of the regolith on the Moon is found to be correlated with the effects of space weathering. The agents of space weathering alter the physical and chemical properties of the lunar soil. There is a wide range of different types of impactors such as solar wind particles, micrometeorites $(<1 \mathrm{~mm})$, meteorites, solar and galactic cosmic rays (Pieters \& Noble, 2016). When the micrometeorites interact with the regolith, it results in the comminution of the solid materials accompanied by impact melting and rapid vaporization. This finally deposits the oxygen-depleted silica-rich glass (Heiken et al., 1991). On the contrary, the solar wind interaction results in sputtering, irradiation, and implantation producing an oxygen-rich amorphous glass layer (Keller \& Mckay, 1997). However, sufficient energy exists in the micrometeorite impacts to partially or completely melt the soil. This incorporates surrounding glass

\footnotetext{
* Corresponding author
} 
and mineral fragments into glass welded with soil particles forming easily comminuted aggregates called agglutinates. These are quite common in lunar soil with size ranging from few micrometers to few millimeters. Furthermore, the agglutinates appear black to the human eye due to the presence of minute blebs of metallic iron (nanophase iron) (Taylor, Keller, Morris, $\&$ Mckay, 2001). One of the ubiquitous products of space weathering is the spectral change that occurs particularly in the ultraviolet, visible and near-infrared wavelengths. This is primarily due to the inclusion of nanophase iron in both agglutinates and accreted rims on individual grains (Heiken et al., 1991; Noble, Pieters, \& Keller, 2007; Pieters \& Noble, 2016; Taylor et al., 2001). The micrometeorite impacts result in the vaporization of the soil grains due to enormous temperatures reached by some melts (Keller \& Mckay, 1997). Further dissociation of these molecules into charged ions of elements takes place based on the temperature profile. This is followed by a subsequent deposition of silica-rich platinas with their myriads of nanophase iron (np-Fe ${ }^{\circ}$ (Keller \& Mckay, 1997).

\subsection{Solar Wind Interaction with Lunar Regolith}

When the incoming energized stream of solar wind particles bombard the irradiated lunar regolith, then the atoms eject from the lattice site of the target material depending on the activation energy of the crystallographic structure, thereby, producing an amorphous layer (Stern, 1999). Such sputtered particles from the lunar soil results in redeposition as a thin film in their ballistic direction. However, the amorphous rims produced by the solar wind have different elemental chemistry as compared to the host crystalline surface (Keller \& Mckay, 1997). Particularly, the cations having the lowest crystalline binding energy get sputtered the most resulting in the depleted silicate rims (Bradley, 1994). These amorphous silicate matrices are most commonly present in ilmenite, with different chemical constituents than the host. In addition to this, the deficit sites also allow the incoming solar wind volatiles to get implanted depending on the surface temperature and activation energy associated with the implantation site.

\subsection{Effects of Solar Wind Flow on Surface Evolution}

The solar wind implanted volatiles are directly correlated with the surface exposure, soil chemistry, and surface temperature. More mature mare soils have a comparatively higher exposure time towards solar wind bombardment and hence, constitutes a much greater enrichment of the implants within the agglutinates (Heiken et al., 1991; A. Taylor et al., 2001). This implantation rate depends on the size of the grains and is more in the case of glass welded aggregates produced by micrometeorite impacts (DesMarais, Hayes, \& Meinschein, 1974). Moreover, a lunar regolith grain has an average time span of $10^{3}$ years towards the repeated exposure of solar wind over different time scales (Poupeau, Michel-Levy, Mandeville, \& Romary, 1978). Regarding the surface mineralogy, ilmenite $\left(\mathrm{FeTiO}_{3}\right)$ retains solar wind implants in a much better way than any other mineral. It contains as much of 10 to 100 times of the ${ }^{3} \mathrm{He}$ than that of any other mineral (Heiken et al., 1991). Solar wind proton implantation process has been widely used for examining the formation of $\mathrm{OH}$ bond from the solar wind interaction in the regions with oxide-rich silicate layers (McCord et al., 2011). In addition to this, during the time when the Moon is fully shielded by the Earth's Magnetosphere, some of the oxygen ions originating from the Earth's upper atmosphere are carried by the solar wind to strike the lunar surface at relatively lower speeds. This attributes to an increased amount of lunar oxygen repository across the regolith. Furthermore, the surface temperature affects the brittleness and overall crushability of the soil grains resulting in melting and formation of agglutinates (Paige et al., 2010). However, the diurnal variations of extreme lunar temperatures show no significant effect on mechanical weathering of lunar soil. This illustrates a higher concentration of solar wind particles for finer soil grains which are correlated with higher surface areas. This study focusses on investigating the interaction of solar wind with the lunar regolith by incorporating a simulation-based approach for quantitatively assessing the retention and reflection of the solar wind implanted volatiles. An attempt has been made to associate the spatial characteristics of the retained ${ }^{3} \mathrm{He}$ by analyzing the textural patterns and its frequency domain. The study also investigates the possibility of lunar oxygen production.

\section{IMPLANTATION OF SOLAR WIND VOLATILES}

When the solar wind interacts with the lunar regolith, about $1 \%$ of the incoming protons get reflected back into the solar wind (Saito et al., 2008). However, this reflection rate increases to $50 \%$ at the magnetic anomalies (Lue et al., 2011; Poppe, Halekas, Delory, \& Farrell, 2012). Observation also suggests that there may be $2-4 \%$ probable chance of ${ }^{3} \mathrm{He}$ interacting with the surface along with very minute traces of heavier ions. Apart from this, for some particular cases where the test surface contains high levels of ilmenite-rich pyroclastic deposits, the implanted proton (in the form of neutral hydrogen when charge exchange with the surface occurs) tend to reduce ilmenite to form $\mathrm{OH}$ bond by liberating lunar oxygen (Taylor \& Carrier, 1992). The surface temperature also plays an important role in regulating the retention and reflection rates of a particular implant.

\subsection{Proton Implantation into Lunar Regolith}

Temperature variations applicable to equatorial lunar surface cause the neutral $\mathrm{H}$ to be produced by the exchanging of protons with the surface (Sunshine et al., 2009). The diffusion/retention rates span many orders of magnitude with ranges varying from $10^{-2} \mathrm{~s}$ in warm regions to $10^{15} \mathrm{~s}$ in cold traps. Low activation energy generally less than $0.2 \mathrm{eV}$ attributes to a crystallographic defect-free region and sometimes, a region with a vertical channel defect. In situations like this, the incident protons are immediately diffused by the crystal back into space as a neutral $\mathrm{H}$ within $30 \mathrm{~s}$. These surfaces are generally associated with low maturity signifying less exposure to the space environment. Such immature fresh material could be regarded as $\mathrm{H}$ emitting surface. In contrast, there are regions which have high activation energy with large numbers of vacancies in the crystal lattice. These vacancies are often created by the continuous bombardment of space weathering agents signifying a more matured soil with agglutinates as primary products. At times, there exist hole-type defects which often makes it difficult for the incident solar wind proton to migrate. This case significantly increases the diffusion rate by a month or so even for the regions offering warmer temperatures. Hence, the materials with high activation energy normally offer a retentive surface for $\mathrm{H}$. However, the intermediate surfaces with median activation energies correspond to a contrasting nature of the crystal. This case is heavily dependent on surface temperature. When temperatures are above $300 \mathrm{~K}$ (i.e. warm regions), the surface acts as an emitter for any of the incident protons on timescales less than a second, Hence, any $\mathrm{H}$ normally gets emitted after the charge exchange of proton with the surface. On contrary, for the colder regions with temperatures going down below $150 \mathrm{~K}$, the surface behaves retentive to any of the $\mathrm{H}$ thereby increasing the diffusion time more than a lunation. Moreover, this process of retention continues until the surface rotates into higher temperature regions when the crystallographic lattice gets energized again to emit the 
$\mathrm{H}$ back into space. Such diurnal variations caused by the intermediate activation energy could also be observed in (Sunshine et al., 2009).

Several analysis indicated the presence of defects in the top 100 $\mathrm{nm}$ of the lunar regolith mainly due to the alterations made in the lattice of the exposed rims of the lunar grains by the space environment (Keller \& Mckay, 1997; Noble, Keller, \& Pieters, 2010; Noble et al., 2007; Pieters \& Noble, 2016; Taylor et al., 2001). Apart from this, some rims consist of destroyed lattice mainly due to continual solar wind penetration, while there are instances where the rims mark inclusions of nanophase iron due to the sputtered or impact vapor deposition (Keller \& Mckay, 1997; Noble et al., 2010). This correlates with the fact that these exposed lunar soil grains are characterized with crystal defects ranging from vacancies, channels to crystal destruction (Keller \& Mckay, 1997).

\subsection{Constraints for Solar Wind Implanted ${ }^{3} \mathrm{He}$}

Among all the solar wind implanted volatiles, ${ }^{3} \mathrm{He}$ remains the most valuable resource for its potential of being used as a fusion fuel. Contrary to the fact that Earth has its own magnetic field, the lunar surface has been subjected to large quantile bombardment of ${ }^{3} \mathrm{He}$ by the solar wind. Eventually, up to $\sim 20$ ppb (by weight) of ${ }^{3} \mathrm{He}$ is being implanted in some lunar soils by the irradiation of the lunar regolith by the solar wind. Due to its non-radioactive nature, implanted ${ }^{3} \mathrm{He}$ could provide safer nuclear energy in a $\mathrm{D}-{ }^{3} \mathrm{He}$ fusion reactor, thereby satisfying the energy needs of the Earth (Santarius, Kulcinski, Miley, 2006; Santarius, 2004). The concentration of ${ }^{3} \mathrm{He}$ in the lunar regolith at a given location is mainly governed by two factors: the amount of ${ }^{3} \mathrm{He}$ implanted by the solar wind and efficient retention of implanted ${ }^{3} \mathrm{He}$, i.e. soil outgassing (Fa \& Jin, 2007). Soil outgassing includes optical maturity (OMAT), which amounts to the exposure time of the lunar surface to the space environment and ilmenite abundance, capable of retaining ${ }^{3} \mathrm{He}$ reserves (Heiken et al., 1991; Lucey, David, \& Hawke, 1998; Lucey, Blewett, Taylor, \& Hawke, 2000; Shukla, Kumar, \& Agrawal, 2017). $\mathrm{TiO}_{2}$ serves as a good tracer of ilmenite concentration. It is noteworthy that the ${ }^{3} \mathrm{He}$ content shows a high correlation with the product of maturation and ilmenite abundance for the Apollo samples (Conway, 1988; Jordan, 1990). Moreover, it takes around $\sim 10^{3} \mathrm{y}$ for a regolith grain to reside on an exposed lunar surface to the solar wind which eventually increases to $\sim 10^{7}-10^{8}$ y for a typical surface exposure to cosmic rays (Poupeau et al., 1978).

\subsection{Implications of Interplanetary Coronal Mass Ejection on Solar Wind Implantation Process}

Interplanetary Coronal Mass Ejections are large explosions of plasma and magnetic field from the Sun's upper atmosphere, Corona. The strength of interplanetary magnetic field in the case of ICME is far more than that of the nominal solar wind, ejecting billions of tons of coronal materials (Burkepile, Hundhausen, Stanger, Cyr, \& Seiden, 2004; Halekas, Bale, Mitchell, \& Lin, 2005; Killen et al., 2012). The major source of such CMEs is usually the region of the Sun having strong and stressed localized fields of magnetic flux, such as an active sunspot group. These are sometimes associated with flares but can occur independently. During this solar activity, the speed significantly increases twice that of the normal case with the density being 10 times (Halekas et al., 2005; Killen et al., 2012). It has been shown that the yield of heavy ion component especially ${ }^{3} \mathrm{He}, \mathrm{O}^{7+}$ can heavily increase the total implantation into the lunar regolith during the times of enhanced population of heavy ions in CMEs
(Killen et al., 2012). Furthermore, the simulation results indicated an abrupt increase of the mass of $\mathrm{Na}, \mathrm{K}, \mathrm{Ca}$ and $\mathrm{Mg}$ in the lunar exosphere more than ten times the background values. This attributes an escalated probability of sputtered neutral particles for escaping the regolith (Killen et al., 2012). However, the frequency of CME varies with the sunspot cycle. During the time of solar minimum, one CME is observed per week which eventually increases to two or three at the solar maximum (Burkepile et al., 2004; Halekas et al., 2005; Lin \& Forbes, 2000).

\section{MODEL DESCRIPTION}

In order to evaluate the evolution of the solar wind interaction with the lunar surface, a computer simulation for understanding the implantation process has been incorporated. The study considers an exposed surface (to solar radiation and solar wind) that has been reconstructed as a function of local time $\left(\mathrm{T}_{\mathrm{L}}\right)$. Specifically, an equatorial patch of regolith is divided into twelve discrete segments wherein the temperature $\mathrm{T}$ (measured in Kelvin) and solar wind flux S are defined as (1) (Farrell, Hurley, \& Zimmerman, 2015):

$$
\begin{gathered}
T=280 \cos ^{0.25}(\theta)+100 \\
S=n_{s w} v_{s w} \cos (\theta)
\end{gathered}
$$

In the above equation,

$$
\begin{aligned}
& \theta=\frac{12-T_{L}}{24}, T_{L} \in[0,12] \text { and } \theta \in[0,2 \pi] \\
& n_{s w}: \text { number of particles in one unit of volume of regolith } \\
& v_{s w}: \text { velocity of the solar wind }
\end{aligned}
$$

Statistically, the activation energy $(\mathrm{U})$ for each regolith particle has been assumed to be in random normal distribution. Smaller values of $U$ attribute to a regular defect-free crystal structure (immature) while larger bandwidths signify the presence of an increased number of crystal defects (mature). The assigned $U$ values along with the calculated $\mathrm{T}$ and $\mathrm{S}$ from (1) initiate the simulation predicting the probable regions of the presence of solar wind implanted volatiles. Further, the density and velocity of the solar wind are set randomly in a way that it varies continuously for every hour of local time starting from dawn.

Normally, there are two challenging effects for the dayside lunar surface. The former increases the solar wind incident flux as the surface approaches to local noon, while the latter increases the surface temperature towards the local noon. More to this, if the surface holds high $U$ values then it behaves as a retaining surface. This allows the low and intermediate $U$ value distributed surface to emit the $\mathrm{H}$ and minute traces of heavy ions, thereby, creating an emitting surface as noon is approached. In the present study, the simulation has been carried out for a month considering the effects of ICME and complete blackout on the implantation process. The simulation also incorporates the notion of reduced nighttime plasma concentration on the lunar regolith. During this phase, a plasma void is created which attributes to approximately 500 times reduction of the solar plasma as compared to that of the dayside (Burkepile et al., 2004; Farrell et al., 2015; Halekas et al., 2005; Killen et al., 2012). Moreover, it has been observed that when the Moon is completely shielded from solar wind bombardment by the Earth's magnetosphere, biogenic terrestrial oxygen is transported (at least $2.6 \times 10^{4}$ ions $\mathrm{cm}^{-2} \mathrm{~s}^{-1}$ ) from the Earth and implanted into the lunar regolith (Terada et al., 2017). This phenomenon has also been modeled in this work, which accounts for the total lunar oxygen repository. Apart from this, the model also assimilates the frequency domain analysis along with textural characterization of the solar wind implanted ${ }^{3} \mathrm{He}$. 


\section{SIMULATION RESULTS}

In this study, the evolution of solar wind implanted volatiles has been simulated by traversing $5 \times 10^{6}$ test particles with a velocity of $\sim 400 \mathrm{~km} / \mathrm{s}$ over a reconstructed grid surface size of $100 \times 100$ (assuming $1 \mathrm{~m}$ to be the spatial resolution of the cell). The surface temperature varies as a cosine function of the local time and is represented as the distribution of hourly exposed simulated regolith (in Figure 1).

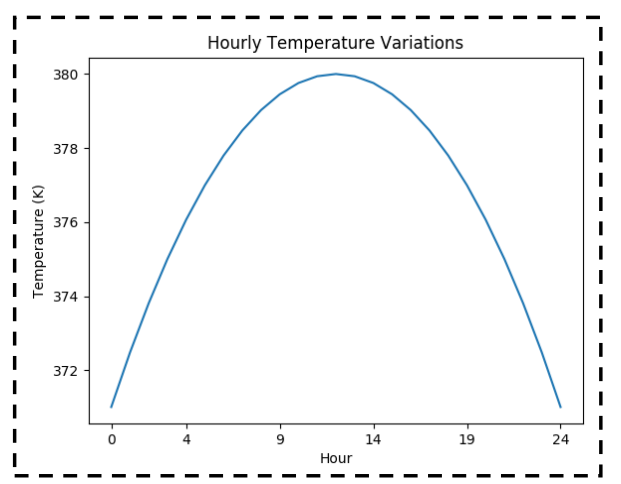

Figure 1. Simulated surface temperature (in $\mathrm{K}$ ) for an equatorial patch of the lunar regolith.

The minimum temperatures of the simulated surface are observed during the midnight, with the peak noon temperature of around $380 \mathrm{~K}$. This simulation emphasizes mid-latitude equatorial regions while undermining the polar patches where the temperatures could go below $100 \mathrm{~K}$ for permanently shadowed regions. The reason being the non-uniformity of the solar wind ion flux at the crater floors possessing ambipolar evolution (Zimmerman, Farrell, Stubbs, Halekas, \& Jackson, 2011). Also, the flux level degrades by a varying factor of 10-100 as compared to that of the direct incidence. In order to evaluate the rate of maturation of the lunar regolith, the distribution of activation energy for each grid cell has been randomly generated. During the initial setup of the model, it is assumed that the dissemination of the values over each line of the grid follows a Gaussian distribution. However, in real time, the existing crystallographic defects tend to follow a more complicated distribution which may be exhibited in a combination of Gaussians as used here. The spread of these activation energies determines the true nature of the soil for carrying out the implantation process when the solar wind ions actually interact with the surface. These ions are forced to move with a varying speed of $300-400 \mathrm{~km} / \mathrm{s}$ for a better insight into initiating the simulation. Depending on the scenario as explained in the previous sections, the ions bombard the grid following sputtering, irradiation, and implantation. This simulation is performed for 30 days while incorporating the effect of magnetosphere shielding of the Moon.

For understanding an enhanced variability of the solar wind, a two-day passage of ICME is considered to occur within this month. However, such solar activity eventuates once in three years during strong, long duration solar flares and filament eruptions. These solar wind volatiles incident the surface resulting in either getting retained or released depending on the distribution of activation energy, amount of ilmenite present and surface temperature. The model runs the implantation algorithms as proposed in Section 2. The composition of solar wind reveals a higher proportion of $\mathrm{H}$ with a much decreased varying amounts of heavier trace ions like ${ }^{3} \mathrm{He}, \mathrm{O}^{7+}$ etc. In this preliminary work, all these cases have been randomly simulated based on their proportions.

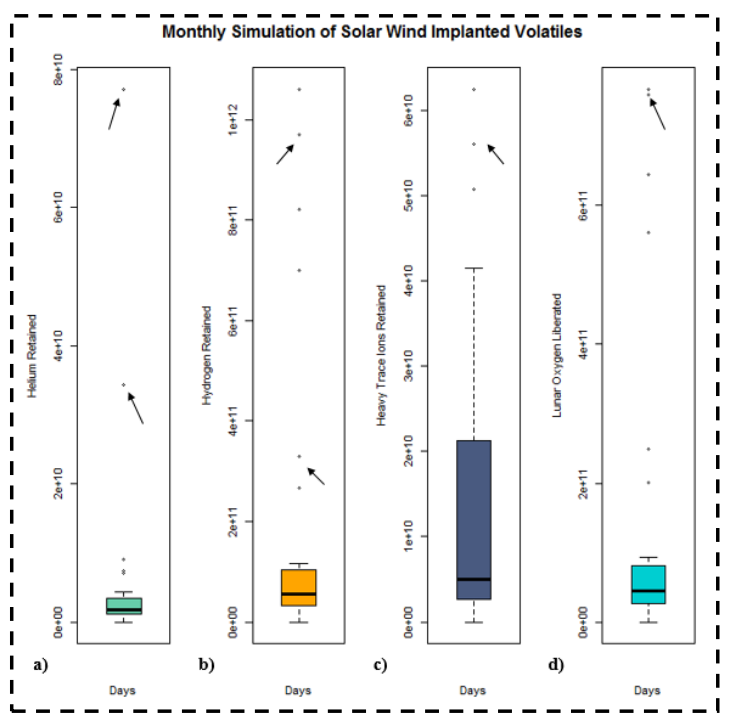

Figure 2. Simulated retention of Solar wind volatiles a) ${ }^{3} \mathrm{He}, \mathrm{b}$ )

$\mathrm{H}, \mathrm{c})$ Trace heavy ions and d) Lunar oxygen liberated.

Each cell of the grid receives a different amount of solar wind particles signifying the effect of topography for the incident stream of charged particles. Importantly, earlier case studies concentrated more on the implantation probability of the protons, charge exchanging with the surface to embed neutral $\mathrm{H}$ ion into the crystal vacancies, thereby, forming the $\mathrm{OH}$ veneer in the oxide-rich regolith layers (Farrell et al., 2015; Feldman, 1998; Saito et al., 2008; Schmitt, Kulcinski, Santarius, Ding, Malecki, $\&$ Zalewski, 2000). However, the present study examines all the cases including the probable implantation of heavier trace ions and correlates the production of lunar oxygen with the $\mathrm{H}$ retained sites. Figure 2 shows a quantitative aspect of the total retention of implants with respect to the number of days the simulation has run. The arrow marks in all the cases are the outliers of the box plots attributing to a much higher proportion of retention due to an increased incident solar flux during a two day ICME. The outliers, in the case of lunar oxygen, also incorporates the transportation of the biogenic terrestrial oxygen from the Earth during the blackout days. This is indicated by an increased number of oxygen atoms in the lunar exosphere during full shielding from the solar wind. The above graphs use a box plot to significantly describe the shape of the distribution, its median, and variability. Here, the increased variability in the case of retained heavier trace ions possesses a difficulty in modeling the impact of the activation energy of the lunar soil on these.

In this study, an attempt has been made to synthesize the retained mechanism in order to correlate the amount of implanted species (especially heavier trace ions) with each of the local time segment. Due to the decreased proportion of protons during the time of ICME, the distribution of neutral $\mathrm{H}$ ions by surface charge exchange lessens occasionally as compared to that of the normal days (Figure 3). Correspondingly, a large number of implantations at the local noon attribute to a prolonged loitering of these ions resulting in their lower diffusion rate as compared to that of the impact vaporization processes (Farrell et al., 2015). Upon determining the rate of change in the hourly implanted Hs, nearly $6 \%$ of the incoming Hs are retained for longer periods as the time approaches the local noon. However, this percentage increases to $9 \%$ during a 2 -day ICME passage. It has also been observed that the retained $\mathrm{Hs}$ are within the saturation limits of $\sim 5 \times 10^{28} / \mathrm{m}^{3}$ in the top $100 \mathrm{~nm}$ of exposed regolith (Starukhina, 2001). 


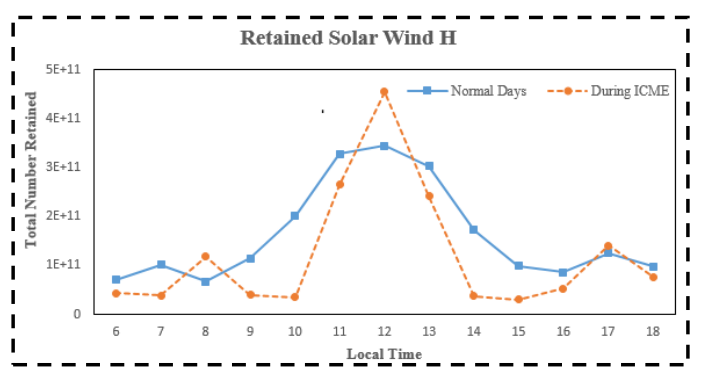

Figure 3. Comparison of $\mathrm{H}$ ions retained during ICME and that of the normal days.

Normally, the distribution of low activation energy values attributes to a surface with no defects making it a suitable escaping site for neutral $\mathrm{H}$ ions. Such cases allow no loitering of the $\mathrm{H}$ to significantly create $\mathrm{OH}$. Figure 4 represents a decreasing trend in retained implantations of $\mathrm{H}$ and heavier trace ions at local noon due to an escalated temperature profile of the regolith. This also correlates with the larger proportion of particles having the intermediate $U$ values resulting in faster diffusion in warmer regions. However, the lower temperatures tend to retain the volatiles resulting in the formation of $\mathrm{OH}$ in the oxide rich regolith. This diurnal behavior of surface temperature is well documented for realizing the temporal and spatial variability of lunar hydration (Sunshine et al., 2009). The slope of the Vshaped loitering $\mathrm{H}$ profile moderately increases for the intermediate activation energy range during the time of ICME, while it almost negates when the Moon is completely covered by the Earth's magnetosphere.

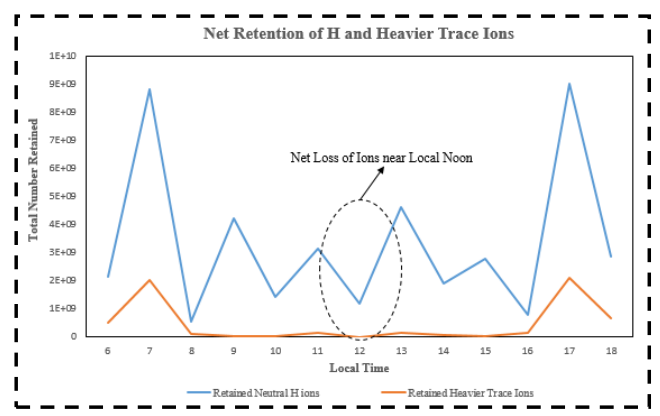

Figure 4. Total retained $\mathrm{H}$ and heavier trace ions as a function of $T_{L}$. This describes the scenario of the simulated lunar regolith with some defects which correlates with the intrinsic mineralogy responsible for crystal strength.

The distribution of larger activation energies, usually greater than $1 \mathrm{eV}$, attributes to a surface with many defects. As evident in Figure 5, the diffusion of $\mathrm{H}$ and trace heavy ions has not been observed at local noon. Rather, the increased proportion of $\mathrm{H}$ ions being retained from $8 \mathrm{~h} T_{L}$ to noon $T_{L}$ is offset by a factor of incident solar wind flux having temporal variations. Similar behavior has been replicated by the implantation of the significantly lower proportion of trace heavy ions (like $\mathrm{O}^{7+}$ ). In order to better visualize and compare the concentration of the retained particles for both the cases, the curve signifying heavy ions is multiplied by a scale factor of 15 . In this regard, the mild noon emissions created by local temperatures are negated due to the large incident solar wind flux interacting with the simulated surface. Moreover, during ICMEs, the density of the solar wind increases by ten times thereby reconstructing this scenario of creating much more loitering of $\mathrm{H}$ and heavy ions implantations in particular (in Figure 3).

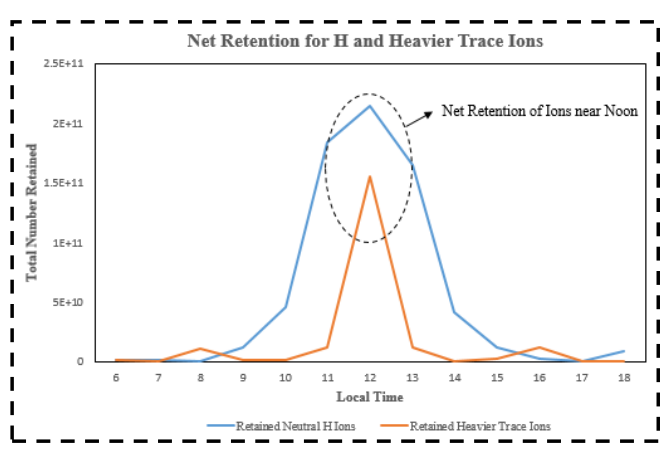

Figure 5. Total retained $\mathrm{H}$ and heavy trace ions as a function of $\mathrm{T}_{\mathrm{L}}$. The retained number of heavy ions is multiplied by 15 in order to visualize with a comparatively higher proportion of $\mathrm{H}$

ions. There are no local minima at noon due to the large population of particles with high activation energy $(\sim>1 \mathrm{eV})$.

Further, the increased proportions of heavy ions bombard the surface creating a richer set of defects in the agglutinate material. However, other effects such as long-term gardening and soil reset have not been considered for this study but could be dealt in a more comprehensive manner by integrating remote spectroscopy of lunar rocks as well as Apollo lunar samples. For the range of regolith particles holding very low activation energy values (due to a relatively lesser exposure of materials at the surface), the volatiles simply gets sputtered, transforming the physical and chemical properties of the airless bodies. About $11.2 \%$ of the total particles incident on the regolith for the entire simulation is found to be ejected (diffused) resulting from the immature regions near fresh crater or on steep slopes. Sputtering further attributes to the net loss of photolyzed water from the oxygenrich regolith.

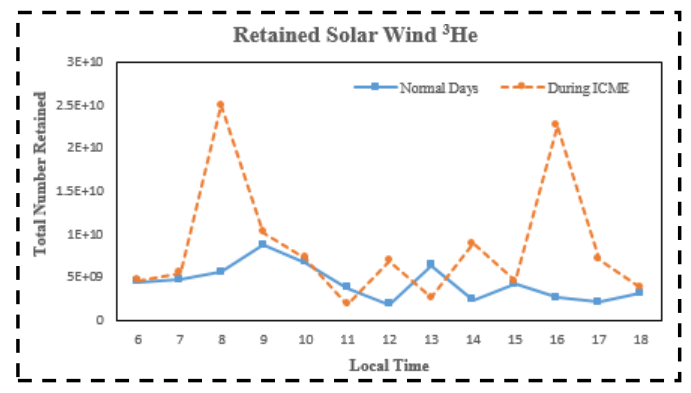

Figure 6. Total retained ${ }^{3} \mathrm{He}$ during ICME and that of the normal days. A net higher retention is observed during ICME.

Along with other ions contained in the solar wind, the present study also examines the implantation scenario of $2-4 \%{ }^{3} \mathrm{He}$. The simulation results in an enhanced population of ${ }^{3} \mathrm{He}$ implants during the 2-day ICME. This amount significantly decreases for normal days as shown in Figure 6. It also proclaims a comparatively lesser retention of exospheric helium at the local noon while the distribution tends to increase during the time of dawn and dusk. The results also provide insights into exploring the temporal variability of the alpha flux responsible for deriving the dominance of helium exosphere. In order to demonstrate the simulation analysis for future lunar mining ${ }^{3} \mathrm{He}$ sites, the present study also incorporates transforming of the spatial domain into the frequency domain to determine probable regions comprising of helium deposits. This work utilizes a Fast Fourier Transform (FFT) algorithm for sampling the surface over a time span and disintegrating it into frequency components (sinusoidal and cosine). 


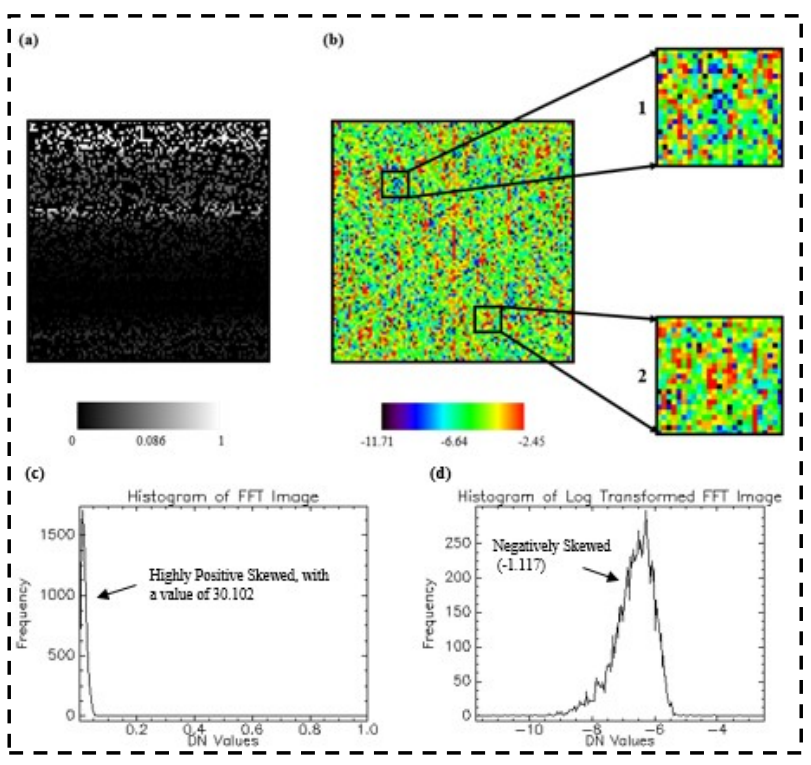

Figure 7. (a) Original simulated spatial domain ${ }^{3} \mathrm{He}$ retained image, (b) Log transformed FFT image, (c) Histogram of FFT image before power transformation, and (d) Histogram of LPTFFT image, with modal abundance and variance of -6.25 and 0.44 respectively.

The reason for choosing this algorithm is to compute the image variability, thereby, evaluating the potential outliers (Heideman, Johnson, \& Burrus, 1984). The generated spatial domain image of the simulated ${ }^{3} \mathrm{He}$ retention is shown in Figure 7 (a), where brighter shades represent a high probability of retention. Figure 7 (b) represents the result of the FFT operation, wherein zone 1 signifies potential ${ }^{3} \mathrm{He}$ retention hotspots (low variance). Figure 7 (a) represents the result of the FFT operation, wherein darker shade (blue to black color) signifies potential ${ }^{3} \mathrm{He}$ retention hotspots showing low variance with a major distribution as shown in zone 1. Similarly, zone 2 denotes high variability attributing towards low probable ${ }^{3} \mathrm{He}$ retaining sites illustrated by yellow to red color. The corresponding histogram obtained by applying subsequent log power transformation on the FFT image as shown in Figure 7 (d) tends to have a negatively skewed nature having the mean, standard deviation, skewness and kurtosis of $6.648,0.663,-1.117$ and 3.704 respectively. This high excess kurtosis signifies heavily tailed leptokurtic distribution. As evident in Figure 7 (c), the FFT histogram being highly skewed, a Log Power Transformation (LPT) on the magnitude of the frequency domain complex values has been taken to reduce the skewness and make the data more interpretable. Since the magnitude lies in between 0 and 1 (both exclusive), the resulting DN values are negative. The mathematical equations for the aforementioned transformations are given in (2) and (3) (Heideman et al., 1984).

$$
\begin{aligned}
& F(u, v)=\frac{1}{M N} \sum_{m=0}^{M-1} \sum_{n=0}^{N-1} f[m, n] e^{-j 2 \pi\left(\frac{u}{M} m+\frac{v}{N} n\right)} \\
& L(u . v)=10 \log _{10}(|F(u, v)|)
\end{aligned}
$$

where,

$F(u, v)$ : FFT image of $\mathrm{M}$ rows and $\mathrm{N}$ columns. $f[m, n]$ : Spatial image with the pixel value at $[\mathrm{m}, \mathrm{n}]$ location. $L(u, v)$ : Log transformed image.

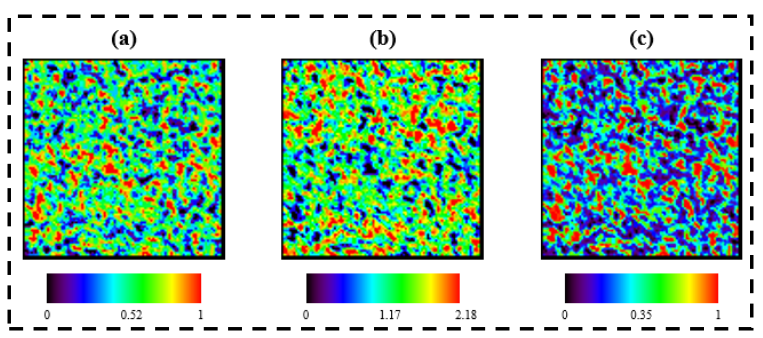

Figure 8. Textural parameters of the retained ${ }^{3} \mathrm{He}$. (a) IDM, (b) Entropy, and (c) ASM.

Furthermore, several second-order statistical measures of the original image are estimated for quantitatively assessing the simulated image texture. This is performed by incorporating the Gray Level Co-occurrence Matrix (GLCM) and is shown in Figure 8. In the Inverse Difference Moment (IDM) image, it has been observed that the zones representing high probable ${ }^{3} \mathrm{He}$ retention display uniform surface isotopic composition attributing to the heterogeneous nature of the lunar terrain. However, the red patches could possibly indicate smooth mare regions comprising of crater fills with little variations in the topography (as shown in red color in Figure 8 (a)). The entropy image provides the measure of disorderliness. As evident in Figure 8 (b), the patches that are characterized by increasing values of entropy may exhibit poor ${ }^{3} \mathrm{He}$ retention capabilities. The inverse operation of the entropy analysis is the Angular Second Moment (ASM) which calculates the textural uniformity of the simulated image. The red regions, in Figure 8 (c), could indicate areas having a higher probability of retaining ${ }^{3} \mathrm{He}$. The overall textural analysis concludes that the simulated results conform to the previous studies showing that the maximum ${ }^{3} \mathrm{He}$ ions are retained in the homogenous mare fills, attributing to the implications of conducting future lunar mining operations in these regions (Conway, 1988; Fa \& Jin, 2007; Farrell et al., 2012).

As part of the lunar oxygen simulation study, the variability of the transported biogenic terrestrial oxygen from the Earth to the lunar surface has been kept in the range [2.6E+4, 3E+4], which is shown in Figure 9. These ions are highly charged as compared to the oxygen ions liberated during the reduction of ilmenite by solar wind implanted $\mathrm{H}$ ions. However, the amount of $\mathrm{O}^{+}$ transported particles is lower than that of the amount liberated. In the simulated scenario, only $0.3 \%$ of the total lunar oxygen content is due to the Earth transported oxygen whereas the remaining $99.7 \%$ ions constitute liberated oxygen ions from $\mathrm{H}_{2} \mathrm{O}$. This is also reflected in the box plot analysis in Figure 2 (d) where the liberated $\mathrm{O}_{2}$ is within the $10^{11}$ range.

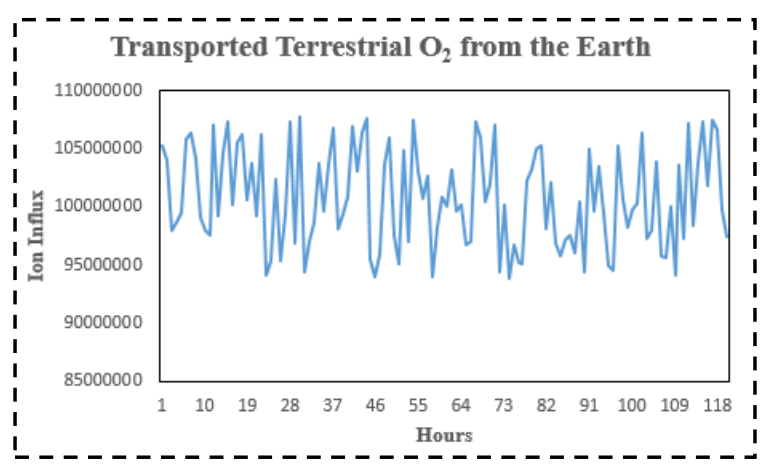

Figure 9. Simulated transported $\mathrm{O}_{2}$ from the Earth to the lunar surface during solar blackout days. 


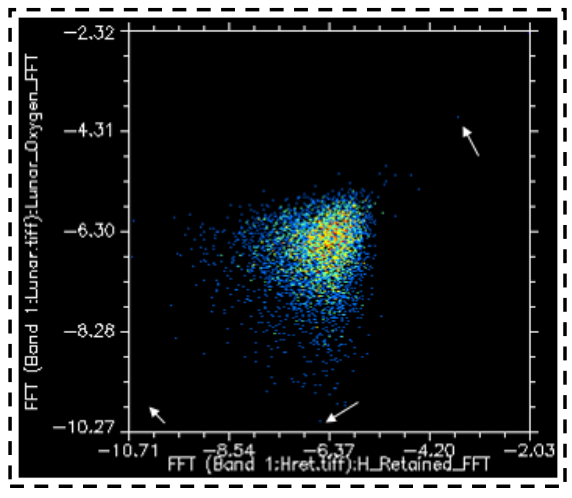

Figure 10. Scatter plot of LPT-FFT images, signifying the relationship of liberated lunar oxygen with the retained $\mathrm{H}$ sites.

Moreover, the total lunar oxygen repository (consisting of both transported $\mathrm{O}^{+}$and oxygen generated from the reduction of $\mathrm{FeTiO}_{3}$ by $\mathrm{H}$ ion) displays an interesting correspondence with the retained hydrogen particles in the lunar regolith. This observation has been made by comparing the log-transformed FFT images using 2D scatter plot as shown in Figure 10. Here, the minute red color patches in the central portion of the distribution arise owing to the oxygen liberated due to ilmenite reduction, i.e. these regions display the highest probability of such an event. This probability, like a point spread function, diminishes as one moves further away from the central dense cloud. The arrow marks denote the outliers attributing to the transported oxygen from the Earth when the Moon lies in the Earth's magnetotail.

\section{CONCLUSION}

In this study, an effort has been made to synthesize the solar wind volatile implantation scenario into the lunar regolith using a simulation-based approach. Although the parameters utilized in this simulation are chosen randomly by incorporating spatiotemporal variability, the results are in concordance with those of the previous works carried out using the Monte Carlo approach (Farrell et al., 2015; Saito et al., 2008). It has been observed that approximately $51 \%$ of the total retained $\mathrm{H}$ particles are used in the reduction reaction of $\mathrm{FeTiO}_{3}$ for liberating lunar oxygen. Significantly, the simulated $\mathrm{H}$ retained sites could be further used to analyze known $2.8 \mu \mathrm{m}$ spectral signature for possible characterization of the regolith containing high hydroxyl content. Similarly, the simulated zones displaying heavy trace ion retention could be utilized for subsequent lunar exospheric studies. The major difference between the proposed model to that of the existing ones lies in the fact that, this study tries to simulate the exact generic scenario for airless bodies (like Moon, Mercury, Asteroids) along with the consideration of the effects of 2-day ICME on the implantation process. Furthermore, the utilization of FFT and textural analysis for exploring potential ${ }^{3} \mathrm{He}$ hotspots is unknown in the previous literature, thereby, showing the uniqueness of this study. In this regard, the study strongly recommends the usage of photoionization effects for accurately realizing the lunar exosphere model.

\section{REFERENCES}

Bradley, J. P. (1994). Nanometer-scale mineralogy and petrography of fine-grained aggregates in anhydrous interplanetary dust particles. Geochimica et Cosmochimica Acta, 58(9), 2123-2134. https://doi.org/10.1016/00167037(94)90290-9.
Burkepile, J. T., Hundhausen, A. J., Stanger, A. L., Cyr, O. C. S., \& Seiden, J. A. (2004). Role of projection effects on solar coronal mass ejection properties: 1 . A study of CMEs associated with limb activity. Journal of Geophysical Research: Space Physics, 109(A3), 1-16. https://doi.org/10.1029/2003JA010149

Cladis, J. B.; Francis, W. E.; Vondrak, R. R. (1994). Transport toward earth of ions sputtered from the moon's surface by the solar wind. Journal of Geophysical Research, 99, 53-64. Conway, J. E. (1988). Lunar Helium-3 and Fusion Power. In Proceedings of a workshop sponsored by the NASA office of Exploration and the Department of Energy Office of Fusion Energy (pp. 1-233).

DesMarais, D. J., Hayes, J. M., \& Meinschein, W. G. (1974). The distribtion in lunar soil of hydraogen released by pyrolysis. Lunar and Planetary Science Conference Proceedings, (February).

Fa, W., \& Jin, Y. Q. (2007). Quantitative estimation of helium-3 spatial distribution in the lunar regolith layer. Icarus, 190(1), 15-23. https://doi.org/10.1016/j.icarus.2007.03.014

Farrell, W. M., Halekas, J. S., Killen, R. M., Delory, G. T., Gross, N., Bleacher, L. V., ... Jackson, T. L. (2012). SolarStorm/Lunar Atmosphere Model (SSLAM): An overview of the effort and description of the driving storm environment. Journal of Geophysical Research E: Planets, 117(10), 1-11. https://doi.org/10.1029/2012JE004070

Farrell, W. M., Hurley, D. M., \& Zimmerman, M. I. (2015). Solar wind implantation into lunar regolith: Hydrogen retention in a surface with defects. Icarus, 255, 116-126. https://doi.org/10.1016/j.icarus.2014.09.014

Feldman, W. C. (1998). Fluxes of Fast and Epithermal Neutrons from Lunar Prospector: Evidence for Water Ice at the Lunar Poles. Science, 281(5382), 1496-1500.

https://doi.org/10.1126/science.281.5382.1496

Halekas, J. S., Bale, S. D., Mitchell, D. L., \& Lin, R. P. (2005). Electrons and magnetic fields in the lunar plasma wake. Journal of Geophysical Research: Space Physics, 110(A7). https://doi.org/10.1029/2004JA010991

Heideman, M. T., Johnson, D. H., \& Burrus, C. S. (1984). Gauss and the history of Fast Fourier Transform. IEEE Acoustics, Speech, and Signal Processing Magazine, 1(October), 14-21.

Heiken, G. H., Vaniman, D. T., \& French, B. M. (1991). Lunar Sourcebook. Cambridge University Press. https://doi.org/10.1017/CBO9781107415324.004

Jordan, J. L. (1990). Mapping Pyroclastic Deposits and Other Lunar Features for Solar Wind Implanted Helium. Workshop on Lunar Volcanic Glasses: Scientific and Resource Potential, (January), 43-45.

Keller, L. P., \& Mckay, D. S. (1997). The nature and origin of rims on lunar soil grains. Geochimica et Cosmochimica Acta, 61(I), 2311-2341. https://doi.org/10.1016/S00167037(97)00085-9

Killen, R. M., Hurley, D. M., \& Farrell, W. M. (2012). The effect on the lunar exosphere of a coronal mass ejection passage. Journal of Geophysical Research E: Planets, 117(3), 1-15. https://doi.org/10.1029/2011JE004011 
Lin, J., \& Forbes, T. G. (2000). Effects of reconnection on the coronal mass ejection process. Journal of Geophysical Research: Space Physics.

https://doi.org/10.1029/1999JA900477

Lucey, P., Blewett, D., Taylor, J., \& Hawke, R. (2000). Imaging of lunar surface maturity. Journal of Geophysical Research: Planets, 105(E8), 20377-20386.

https://doi.org/10.1029/1999JE001110

Lucey, P., David, T., \& Hawke, B. (1998). Mapping the FeO and $\mathrm{TiO} 2$ content of the lunar surface with multispectral imagery. Journal of Geophysical Research, 103(E2), 36793699. https://doi.org/10.1029/97JE03019

Lue, C., Futaana, Y., Barabash, S., Wieser, M., Holmstrm, M., Bhardwaj, A., ... Wurz, P. (2011). Strong influence of lunar crustal fields on the solar wind flow. Geophysical Research Letters, 38(3), 4-8. https://doi.org/10.1029/2010GL046215

McCord, T. B., Taylor, L. A., Combe, J. P., Kramer, G., Pieters, C. M., Sunshine, J. M., \& Clark, R. N. (2011). Sources and physical processes responsible for $\mathrm{OH} / \mathrm{H} 2 \mathrm{O}$ in the lunar soil as revealed by the Moon Mineralogy Mapper (M3). Journal of Geophysical Research E: Planets, 116(4), 1-22. https://doi.org/10.1029/2010JE003711

Noble, S. K., Keller, L. P., \& Pieters, C. M. (2010). Evidence of space weathering in regolith breccias II: Asteroidal regolith breccias. Meteoritics and Planetary Science, 45(12), 20072015. https://doi.org/10.1111/j.1945-5100.2010.01151.x

Noble, S. K., Pieters, C. M., \& Keller, L. P. (2007). An experimental approach to understanding the optical effects of space weathering. Icarus, 192(2), 629-642. https://doi.org/10.1016/j.icarus.2007.07.021

Paige, D. A., Foote, M. C., Greenhagen, B. T., Schofield, J. T., Calcutt, S., Vasavada, A. R., ... McCleese, D. J. (2010). The lunar reconnaissance orbiter diviner lunar radiometer experiment. Space Science Reviews, 150(1-4), 125-160. https://doi.org/10.1007/s11214-009-9529-2

Pieters, C. M., \& Noble, S. K. (2016). Space Weathering on Airless Bodies. Journal of Geophysical Research: Planets, 1865-1884. https://doi.org/10.1002/2016JE005128.Received

Poppe, A. R., Halekas, J. S., Delory, G. T., \& Farrell, W. M. (2012). Particle-in-cell simulations of the solar wind interaction with lunar crustal magnetic anomalies: Magnetic cusp regions.

Journal of Geophysical Research: Space Physics, 117(9), 1-16. https://doi.org/10.1029/2012JA017844

Poupeau, G., Michel-Levy, M. C., Mandeville, J. C., \& Romary, P. (1978). Microcrater and solar-flare track maturation of the lunar regolith. Geochmica et Cosmochimica Acta, (January), 137-155. Retrieved from

http://adsabs.harvard.edu/abs/1978mcvl.conf..137P

Saito, Y., Yokota, S., Tanaka, T., Asamura, K., Nishino, M. N., Fujimoto, M., ... Terasawa, T. (2008). Solar wind proton reflection at the lunar surface: Low energy ion measurement by MAP-PACE onboard SELENE (KAGUYA). Geophysical Research Letters, 35(24), 1-6. https://doi.org/10.1029/2008GL036077
Santarius, John F, Kulcinski, G.L, Miley, G. . (2006). A Strategy for D- 3He Fusion Development. ANS Annual Meeting, Reno, NV, (June), 4-8.

Santarius, J. F. (2004). Lunar 3He and Fusion Power. Fusion Technology.

Schmitt, H. H.; Kulcinski, G. L.; Santarius, J. F.; Ding, J.; Malecki, M. J.; Zalewski, M. J. (2000). Solar-Wind Hydrogen at the Lunar Poles. In Proceeedings of Space 2000: The Seventh International Conference (Vol. 1483, p. 35).

Schoröder, W. (2008). Who first discovered the solar wind?

Space Research Today, 171(C), 34-35.

https://doi.org/10.1016/S1752-9298(08)80013-8

Shukla, S., Kumar, S., \& Agrawal, S. (2017). Mineral Mapping of $\mathrm{FeO}$ and $\mathrm{TiO} 2$ of the Cassini crater using Moon Mineralogy Mapper. In 38th Asian Conference on Remote Sensing (pp. 1$10)$.

Starukhina, L. (2001). Water detection on atmosphereless celestial bodies: Alternative explanations of the observations. Journal of Geophysical Research, 106(E7), 14701. https://doi.org/10.1029/2000JE001307

Stern, S. A. (1999). The Lunar Atmosphere: History, Status, Current Problems, and Context. Reviews of Geophysics, 37(4), 453-491.

Sunshine, J. M., Farnham, T. L., Feaga, L. M., Groussin, O., Merlin, F., Milliken, R. E., \& A'Hearn, M. F. (2009). Temporal and spatial variability of lunar hydration as observed by the deep impact spacecraft. Science, 326(5952), 565-568. https://doi.org/10.1126/science.1179788

Taylor, A., Keller, P., Morris, V., \& Mckay, D. S. (2001). Lunar Mare Soils: Space weathering and the major effects of surfacecorrelated nanophase Fe. Journal of Geophysical Research, 106, 27985-27999.

Taylor, L. a., \& Carrier III, W. D. (1992). Production of Oxygen on the Moon: Which Processes Are Best and Why. AIAA Journal, 30(12), 2858-2863. https://doi.org/10.2514/3.48974

Terada, K., Yokota, S., Saito, Y., Kitamura, N., Asamura, K., \& Nishino, M. N. (2017). Biogenic oxygen from Earth transported to the Moon by a wind of magnetospheric ions. Nature Astronomy, 1(January), 1-5. https://doi.org/10.1038/s41550016-0026

Wieser, M., Barabash, S., Futaana, Y., Holmström, M., Bhardwaj, A., Sridharan, R., ... Asamura, K. (2009). Extremely high reflection of solar wind protons as neutral hydrogen atoms from regolith in space. Planetary and Space Science, 57(1415), 2132-2134. https://doi.org/10.1016/j.pss.2009.09.012

Winske, D., Wu, C. S., Li, Y. Y., Mou, Z. Z., \& Guo, S. Y. (1985). Coupling of Newborn Ions to the Solar Wind by Electromagnetic Instabilities and their Interaction with the Bow Shock. Journal of Geophysical Research, 90, 2713-2726.

Zimmerman, M. I., Farrell, W. M., Stubbs, T. J., Halekas, J. S., \& Jackson, T. L. (2011). Solar wind access to lunar polar craters: Feedback between surface charging and plasma expansion. Geophysical Research Letters, 38(19), 3-7. https://doi.org/10.1029/2011GL048880 